\title{
THE EFFECT OF DDT AND DIELDRIN ON MYELINATED NERVE FIBRES
}

\author{
J. van den BERCKEN \\ Institute of Veterinary Pharmacology and Toxicology, \\ University of Utrecht, Biltstraat 172, Utrecht, The Netherlands
}

J. van den BERCKEN, The effect of DDT and dieldrin on myelinated nerve fibres, European J. Pharmacol. 20 (1972) 205-214.

The effects of the chlorinated hydrocarbon insecticides, DDT and dieldrin, on myelinated nerve fibres of the clawed toad, Xenopus laevis, were studied by recording compound action potentials, action potentials of single nerve fibres, and membrane potentials of single nodes of Ranvier. The effect of DDT $\left(5 \times 10^{-4} \mathrm{M}\right)$ was found to be exclusively on the falling phase of the action potential; either it slowed down the whole falling phase or induced a large negative afterpotential. DDT did not affect threshold voltage, rate of rise and amplitude of the action potential, nor the resting potential. Dieldrin, even in high concentrations $\left(10^{-3} \mathrm{M}\right)$, had no appreciable effect on myelinated nerve fibres.

DDT caused repetitive firing in sensory fibres only. The number of repetitive action potentials increased gradually with increasing amplitude of the negative afterpotential, while the interval between the action potentials decreased. These results indicate that the DDT-induced negative afterpotential is directly responsible for the repetitive activity. The possible mechanisms by which the negative afterpotential could induce a repetitive response were further investigated. It is suggested that the rate of recovery from sodium inactivation plays a major role in this process.

$\begin{array}{lll}\text { DDT } & \text { Motor and sensory nerve fibres } & \text { Negative afterpotential } \\ \text { Dieldrin } & \text { Single nodes of Ranvier } & \text { Repetitive electrical activity }\end{array}$

\section{INTRODUCTION}

The chlorinated hydrocarbon insecticides, DDT and dieldrin, are generally recognized as neurotoxins, which act directly on excitable membranes.

Repetitive activity has long been known to be the most characteristic effect of DDT in the invertebrate nervous system. Although all parts of the nervous system are affected, the initial symptoms of DDTpoisoning, i.e. hyperexcitability, ataxia and tremors, are considered to originate from repetitive activity in the sensory nervous system, causing abnormal reflex activity (Roeder and Weiant, 1946, 1948; Welsh and Gordon, 1947; Yamasaki and Ishii, 1954). Further study of DDT-poisoned cockroach and lobster giant axons has revealed that this repetitive activity is associated with an increase in the negative afterpotential, which is due in turn to prolongation of the peak tran- sient sodium current and a suppression of the steadystate potassium current (Narahashi and Yamasaki, 1960; Narahashi and Haas, 1968; Pichon, 1969). An extensive review of the effect of DDT and other insecticides on excitable membranes, which mainly deals with the invertebrate nervous system, appeared recently (Narahashi, 1971).

Most studies of the action of DDT in mammals and other vertebrates are concerned with the central nervous system and indicate a direct excitatory effect of DDT on the brain, particularly on the cerebellum (Hayes, 1959; Woolley, 1968, 1970; Woolley and Barron, 1968). However, there is also evidence of a pronounced effect of DDT outside the vertebrate central nervous system. In DDT-poisoned rats, there is an abnormally high dorsal root activity, which may produce tremor reflexly (Shankland, 1964), and part of the changes in brain electrical activity is probably 
caused by an increased afferent input (Woolley and Barron, 1968). DDT induces repetitive activity in the sciatic nerve of the rat (Eyzaguirre and Lilienthal, 1949; Shankland, 1964) and of the frog (Shanes, 1951). There is only one study in which DDT was applied to single myelinated nerve fibres. Hille (1968) investigated the action of DDT on frog nodes by means of the voltage clamp technique and found a prolongation of the sodium current similar to that in invertebrate nerve. This also resulted in a large negative afterpotential, but no repetitive activity was observed.

The purpose of the present study was to obtain more information about the DDT-induced repetitive activity in myelinated nerve fibres. Since dieldrin causes similar symptoms of poisoning as DDT, the effect of this insecticide was also studied. In the American cockroach, dieldrin has been shown to affect synaptic transmission but not the axonal membrane (Gianotti et al., 1956; Yamasaki and Narahashi, 1958; Ryan and Shankland, 1971; Wang et al., 1971).

A preliminary report of this study has been published (van den Bercken, 1968).

\section{MATERIALS AND METHODS}

\subsection{Materials}

Male and female specimens of the clawed toad, Xenopus laevis, were used throughout the experiments.

DDT (purified p,p'DDT; 2,2-bis-(p-chlorophenyl)$1,1,1$,-trichloroethane) or dieldrin (99.4\% HEOD; $1,2,3,4,10,10$-hexachloro-6,7-epoxy-1,4,4a,5,6,7,8,8aoctahydro-endo-1,4-exo-5,8-dimethano-naphthalene) was dissolved in ethanol to make up a stock solution. This was squirted forcefully through a No. 27 hypodermic needle into Ringer solution to give a fine suspension. The final concentration of ethanol, which was less than $1 \%$ unless otherwise indicated, was found to have no appreciable effect in control experiments. In a few experiments, acetone, in a maximum concentration of less than $0.1 \%$, was used instead of ethanol.

The composition of the Ringer solution was (in $\mathrm{mM}$ ): $\mathrm{NaCl} 115, \mathrm{KCl} 2.5, \mathrm{Na}_{2} \mathrm{HPO}_{4} 2.15, \mathrm{NaH}_{2} \mathrm{PO}_{4}$ 0.85 and $\mathrm{CaCl}_{2}$ 1.8. The experiments were conducted at room temperature, $20-24^{\circ} \mathrm{C}$.

\subsection{Compound action potentials}

Compound action potentials of the sciatic nerve and some other nerve branches were recorded in the conventional way. Excised nerves were mounted in a moist chamber fitted with silver wire electrodes. Rectangular stimuli of $0.1-\mathrm{msec}$ duration were applied through an RF isolation unit and the action potentials were recorded monophasically by means of an AC-coupled differential amplifier (bandwidth 0.2 $\mathrm{Hz}-10 \mathrm{KHz},-3 \mathrm{~dB}$ ).

\subsection{Action potentials of single nerve fibres}

The sciatic nerve was placed in a small bath of Ringer solution. The distal end of the nerve was desheathed and several single nerve fibres were separated from the nerve trunk over a distance of $2-3 \mathrm{~mm}$ with the aid of sharp needles. Action potentials of these nerve fibres were recorded by means of a suctionpipette electrode (Nelson and Frank, 1964). This electrode consisted of a glass-capillary tube, filled with Ringer solution, drawn to an opening of about $50 \mu$. The pipette was inserted into a Perspex holder with a $\mathrm{Ag}-\mathrm{AgCl}$ electrode, which was mounted in a micromanipulator. Because the pipette fitted exactly into the holder, it was possible to suck up one single nerve fibre by pulling the pipette a little out of the holder. During the recording the pipette-opening, which had been carefully fire-polished to avoid damaging the fibre, was just above the surface of the Ringer solution. Rectangular stimuli of $0.1-\mathrm{msec}$ duration were applied to the proximal end of the nerve by a pair of silver wire electrodes and the stimulus amplitude was augmented until an action potential appeared. If, occasionally, more than one fibre was sucked up, fine adjustment of the stimulus amplitude often made it possible to record activity from one fibre only. The recordings were made through an ACcoupled differential amplifier with a bandwidth of $0.2 \mathrm{~Hz}-10 \mathrm{KHz}(-3 \mathrm{~dB})$. The indifferent $\mathrm{Ag}-\mathrm{AgCl}$ electrode was placed in the bath solution, which was earthed through a silver wire.

\subsection{Membrane potentials of single nodes of Ranvier}

Single myelinated nerve fibres were dissected from the sciatic nerve. The isolated fibre was mounted in a Perspex cell with three pools of Ringer solution separated by two transverse partitions and sealed with silicone jelly. The node under investigation was 
placed in the central pool, in which the normal Ringer solution could be replaced by Ringer solution which contained DDT or dieldrin. Current was passed from one side pool through the isolated node in the central pool and the membrane potentials were recorded between the central pool and the other side pool as described before (van den Bercken, 1969).

In one series of experiments, single nerve fibres were dissected from the ramus cutaneous cruris posterior. This nerve branch innervates the skin over the gastrocnemius muscle and contains mainly sensory fibres (Schmidt and Stämpfli, 1964).

\section{RESULTS}

\subsection{Compound action potentials}

I.p. injection or injection into the toad's dorsal lymph sac of DDT, $25 \mathrm{mg} / \mathrm{kg}$ ( $2 \%$ ethanol suspension in Ringer solution) caused hyperexcitability and convulsions within 1-3 hr, followed later by paralysis and death. The compound action potential of the sciatic nerve excised from a DDT-poisoned animal which showed severe symptoms of poisoning was usually followed by a number of more or less regular potential fluctuations of decreasing amplitude, often superimposed on a distinct negative afterpotential.

These potential fluctuations, apparently caused by repetitive activity in parts of the nerve fibres, also appeared in isolated sciatic nerves which were bathed in Ringer solution to which DDT had been added.
$1 \mathrm{hr}$ of exposure to a concentration of $5 \times 10^{-4} \mathrm{M}$ DDT had a marked effect (fig. 1A, B). In the peroneal and tibial branch of this nerve, the amplitude of the repetitive discharges did not grow to more than about $20 \%$ of the maximum amplitude of the first action potential, even after longer exposure $(24 \mathrm{hr})$ to higher DDT concentrations $\left(10^{-3} \mathrm{M}\right)$. In other nerve branches this ratio was completely different. The sensory branch which innervates the skin over the gastrocnemius muscle (ramus cutaneous cruris posterior) showed much more repetitive activity after $1 \mathrm{hr}$ of exposure to $5 \times 10^{-4} \mathrm{M}$ DDT (fig. 1C) than the motor branch which innervates the head of this muscle (fig. 1D). The DDT-induced repetitive activity was not affected by prior removal of the connective tissue sheath. In other excised afferent nerves, i.c., the rami dorsales and the vagus branches that innervate the lateral-line organs, DDT also induced marked repetitive activity.

In a few experiments, the sciatic nerve was dissected with the Xth spinal roots still attached, after opening the spinal canal by dorsal laminectomy. After treatment with $5 \times 10^{-4} \mathrm{M}$ DDT, the dorsal root showed marked repetitive activity, whereas in the ventral root repetitive activity was completely absent, even after $18 \mathrm{hr}$ of exposure to DDT.

Although injection of dieldrin, $25 \mathrm{mg} / \mathrm{kg}$, caused heavy convulsions, no repetitive activity was observed in sciatic nerves excised from dieldrin-poisoned animals. Likewise, nerves bathed in Ringer solution containing dieldrin (up to $10^{-3} \mathrm{M}$ for $24 \mathrm{hr}$ ) did not show

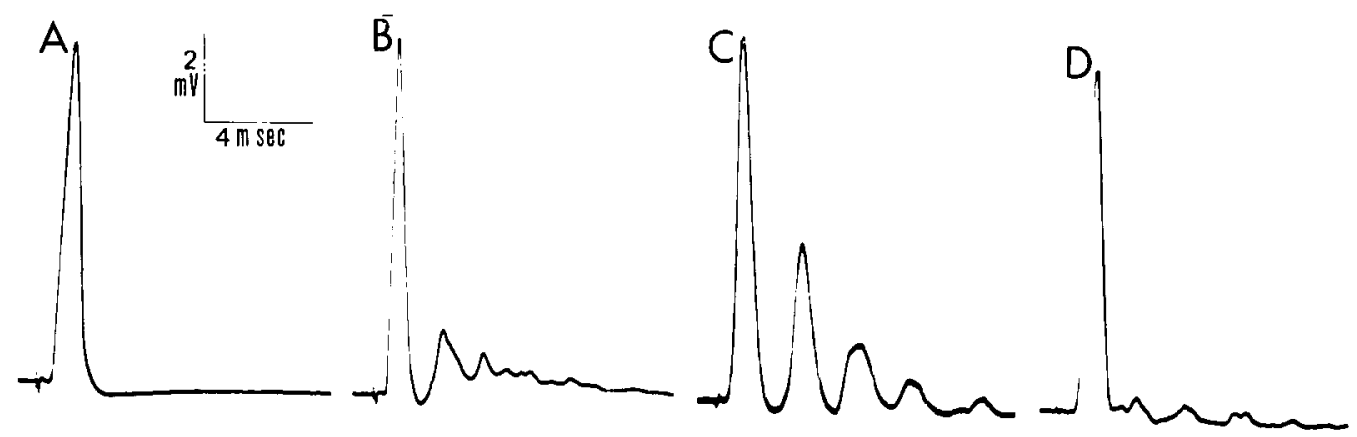

Fig. 1. Effect of DDT on compound action potentials of different branches of the sciatic nerve. Calibration in (A) applies to all records. (A) Compound action potential of the tibial branch before treatment with DDT. The compound action potentials of the other nerve branches have similar shapes. (B-D) Same preparations after $1 \mathrm{hr}$ of exposure to $5 \times 10^{-4} \mathrm{M}$ DDT. Compound action potentials of the tibial branch $(B)$, the ramus cutaneous cruris posterior (C), and of the motor branch to the head of the gastrocnemius muscle (D). 
any sign of repetitive activity. The only effect that could be observed was a slight prolongation of the action potential at the higher concentrations, mainly caused by a slowing-down of the falling phase of the action potential.

\subsection{Action potentials of single nerve fibres}

More information about the DDT-induced repetitive activity was obtained from the experiments with the suction-pipette electrode. With this method, good action potentials of single nerve fibres could be recorded (fig. 2A), although the shape and the amplitude of the spike varied from case to case.

Shortly after the sciatic nerve had been placed in Ringer solution containing $10^{-4}$ or $5 \times 10^{-4} \mathrm{MDDT}$, the action potential was followed, in a number of fibres, by a negative afterpotential (fig. 2B). The amplitude of the negative afterpotential increased progressively with time and attained a level of about $20-25 \%$ of the total spike amplitude within 30-90 min after treatment with DDT had been started. In some of these fibres a repetitive response was observed. At first, a second action potential occasionally appeared, superimposed on the negative afterpotential about $4.5 \mathrm{msec}$ after the first action potential, but soon every stimulating pulse induced two spikes (fig. 2C). Gradually the amplitude of the negative afterpotential increased further and the interval between the two spikes decreased. When this interval reached a period of about $3.5 \mathrm{msec}$, a third action potential appeared, again with a delay of about 4.5 msec (fig. 2D). The negative afterpotential gradually increased while the interval between the spikes decreased until a fourth action potential appeared (fig. $2 \mathrm{E}$ ). Whenever the last interval shortened to about $3.5 \mathrm{msec}$, an additional action potential appeared. Finally, one stimulus induced a train of 7 action potentials, with the interval between the first two spikes as short as $2.3 \mathrm{msec}$ and the successive intervals gradually increasing to about $4.5 \mathrm{msec}$ at the end of the train (fig. $2 \mathrm{~F}$ ). In this particular fibre the height of the spikes remained constant throughout the train. In other fibres, the amplitude of the repetitive action potentials was much lower than the amplitude of the
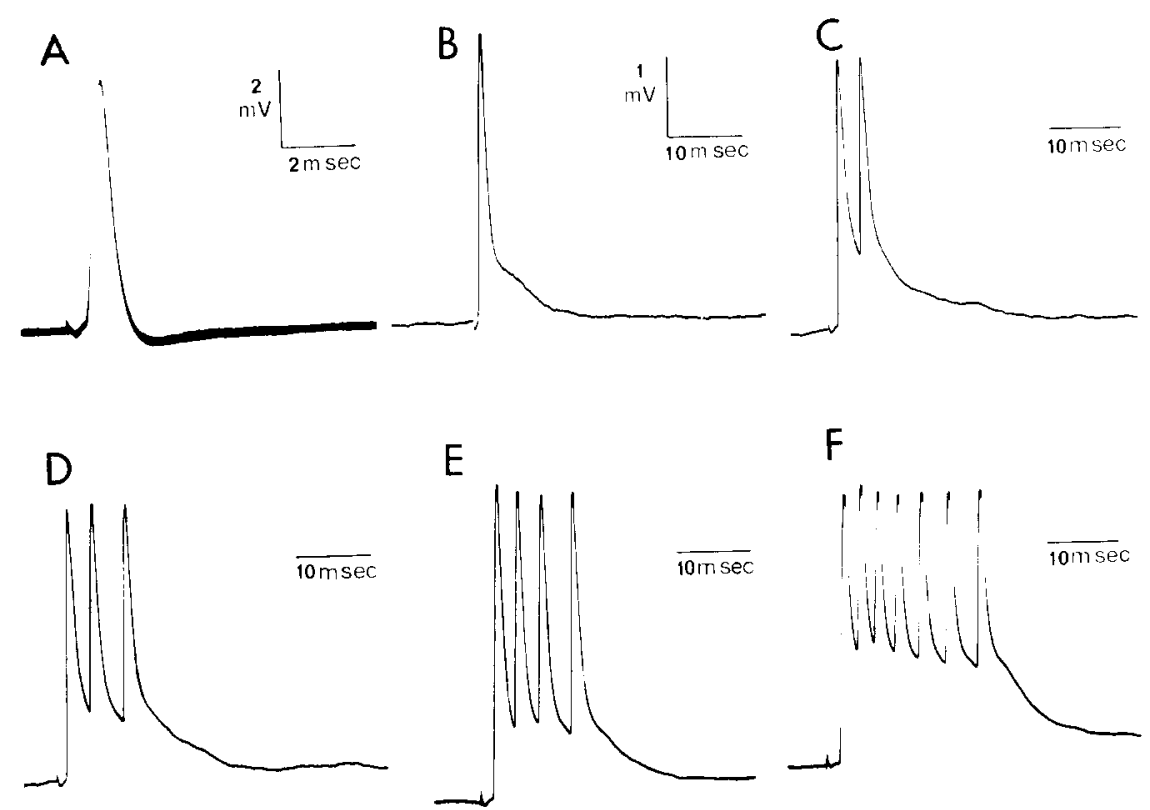

Fig. 2. Repetitive activity in DDT-treated single nerve fibres, recorded with the suction-pipette electrode. Calibration in (B) applies also to records (C-F). (A) Normal action potential of a single nerve fibre, stimulated at a frequency of 10 pulses/sec. In this photograph several oscilloscope traces have been superimposed. (B) Action potential with negative afterpotential, 65 min after start of treatment with $5 \times 10^{-4} \mathrm{M}$ DDT. Stimulus frequency in this and the following records 1 pulse every 2 sec. (C-F) Repetitive action potentials superimposed on the negative afterpotential, after $85,90,105$ and 135 min of treatment with $10^{-4} \mathrm{M}$ DDT respectively. These records were obtained from the same nerve fibre. 


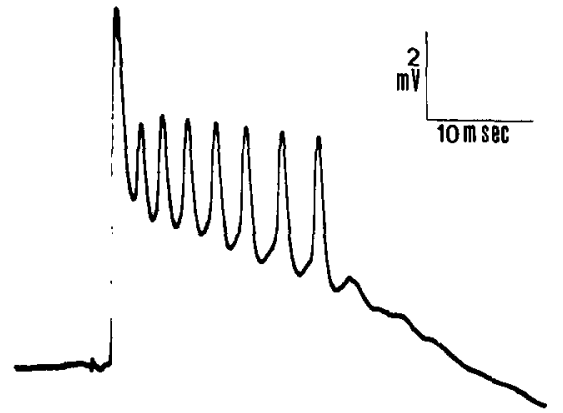

Fig. 3. Repetitive activity in a single nerve fibre, after 195 min of treatment with $5 \times 10^{-4} \mathrm{M}$ DDT. Note the reduced amplitude of the repetitive action potentials.

first action potential (fig. 3). The time it took for DDT to produce a repetitive response varied from fibre to fibre, but in all fibres the amplitude of the negative afterpotential and the number of repetitive action potentials increased progressively with time. The maximum number of repetitive action potentials observed in the individual fibres was usually $4-7$, sometimes 9 or 10 , and in one case 13 . This state of repetitive responding often lasted several hours. In the later stages of poisoning, the number of repetitive action potentials tended to decrease, until finally, $4 \mathrm{hr}$ or more after treatment with DDT had been started, one stimulus induced again one action potential, followed by a long, large negative afterpotential.

Most fibres, however, showed no repetitive activity after treatment with DDT. In about half of these fibres, DDT caused a large negative afterpotential, the amplitude of which often exceeded $25 \%$ of the spike height, yet repetitive firing did not occur. Only small potential fluctuations were sometimes superimposed on the negative afterpotential. In the other fibres, no distinct negative afterpotential was observed and the shape of the action potential remained apparently unchanged. Closer inspection showed, however, that the duration of these action potentials was much longer than normal. These latter effects of DDT were studied further in the experiments with the single nodes. Conduction block attributable to the effect of DDT was not observed in any of the experiments.

In several other series of experiments the sciatic nerve was treated with $5 \times 10^{-4}$ or $10^{-3} \mathrm{M}$ dieldrin, and action potentials of single nerve fibres were recorded. In none of them a negative afterpotential or repetitive firing was observed and the shape of the action potential remained almost normal.

\subsection{Membrane potentials of single nodes of Ranvier}

The action potential of an untreated node of Ranvier was sometimes followed by a small 'undershoot' or positive afterpotential, but usually the falling phase of the action potential passed gradually into the resting potential (fig. 4A). The total duration of the action potential was about $1 \mathrm{msec}$ and depended mainly on temperature.

Action potentials, elicited shortly after the normal Ringer solution bathing the node had been replaced by Ringer solution containing $5 \times 10^{-4} \mathrm{M}$ DDT, were followed by a small negative afterpotential (fig. 4B). The amplitude and duration of the negative afterpotential increased progressively with time, while its time course of decay underwent little change. After 45-60 min of exposure to DDT, the amplitude of the negative afterpotential reached more than $35 \%$ of the total amplitude of the action potential and it took more than $25 \mathrm{msec}$ before the resting potential was restored (fig. 4C). Small, irregular potential fluctuations were often superimposed on the large negative afterpotential. Meanwhile, DDT also caused a marked slowing-down of the initial part of the falling phase, so that the duration of the upper part of the action potential was lengthened to about twice normal, as illustrated by fig. 4D. This figure also shows that DDT had hardly any effect on threshold voltage, rate of rise and amplitude of the action potential, even in the later stage of poisoning. Later on, no more changes occurred, until 2-3 hr after treatment with DDT had been started deterioration of the fibre became apparent. Despite the large negative afterpotential no repetitive activity was observed in these single nodes.

This negative afterpotential was observed in about half the DDT-treated single nodes of Ranvier. In the other nodes, DDT merely caused a marked slowingdown of the whole falling phase of the action potential. The application of $5 \times 10^{-4} \mathrm{M}$ DDT caused a progressive prolongation of the action potential and after 45-60 min of exposure the duration of the action potential was lengthened by a factor of more than 4 , while threshold voltage, rate of rise and amplitude of the action potential again remained unchanged (fig. 4E). 


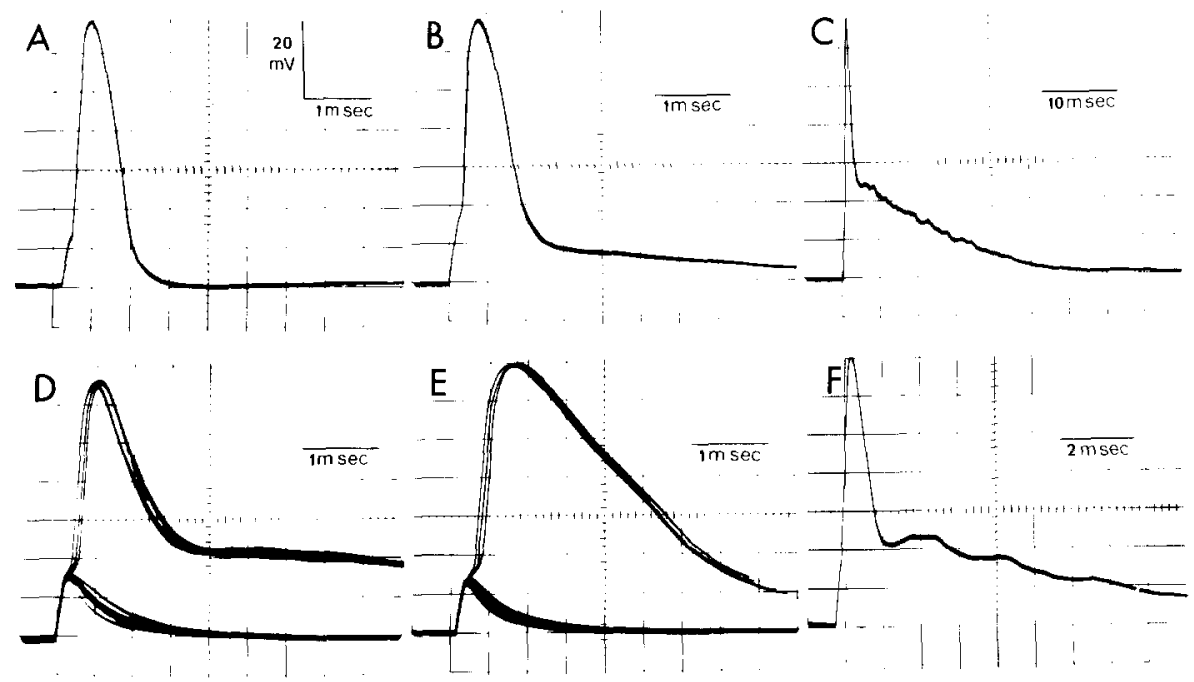

Fig. 4. Effects of DDT on membrane potentials of single nodes of Ranvier. Vertical calibration in (A) applies to all records. (A) Action potential of a single node in normal Ringer solution. (B-D) Action potentials of 3 nodes which developed a large negative afterpotential after treatment with DDT. $6 \mathrm{~min}(\mathrm{~B}), 45 \mathrm{~min}$ (C), and $50 \mathrm{~min}$ (D) after the application of $5 \times 10^{-4} \mathrm{M} \mathrm{DDT}$. (E) Action potential of a node in which DDT caused a prolongation of the whole falling phase, 45 min after the application of $5 \times$ $10^{-4} \mathrm{M}$ DDT. In records (D) and (E) several oscilloscope traces have been superimposed at a near threshold stimulus with a frequency of 2 pulses/sec, showing that the effect of DDT is exclusively on the falling phase of the action potential. (F) Action potential of a single node of a sensory fibre, $8 \mathrm{~min}$ after the application of $5 \times 10^{-4} \mathrm{M} \mathrm{DDT}$.

In a few fibres, an intermediate effect of DDT was observed; together with a marked prolongation of the falling phase of the action potential DDT induced a long negative afterpotential.

In all the nodes investigated, DDT caused no noticeable change in the resting potential (measured as the steady-state potential difference between the central pool and one side pool). The effect of DDT on the action potential was irreversible; no recovery was observed after repeated washing with fresh Ringer solution.

To study the effect of DDT on nodes of sensory fibres, single nerve fibres were dissected from the ramus cutaneous cruris posterior. The dissection of single fibres from this small nerve branch, however, was not very successful, since only 4 out of 10 isolated fibres yielded significant values of action potential. Although DDT caused a large negative afterpotential in these fibres, no repetitive activity occurred. Two differences with respect to the fibres dissected from the sciatic nerve were observed: (1) the negative afterpotential began to develop immediately after application of $5 \times 10^{-4} \mathrm{M}$ DDT and attained its maximum within $20-30 \mathrm{~min}$; (2) the potential fluctua- tions superimposed on the negative afterpotential were more pronounced and appeared as damped oscillations (fig. 4F). Further attempts to dissect single sensory fibres were even less successful.

The time course of decay of the negative afterpotential depended on the resting potential. Hyperpolarization of the nodal membrane, brought about by a long-lasting constant current, caused the negative afterpotential to decay faster, while depolarization had the opposite effect. Occasionally a small depolarization appeared on the afterpotential as the membrane potential was made more negative. This is illustrated by fig. 5 , which shows several afterpotentials at different membrane potentials. The initial level of the negative afterpotential either remained unchanged or increased a little with hyperpolarization and decreased with depolarization.

During the first part of the negative afterpotential, the node was in a refractory state and a short depolarizing pulse induced no active membrane response (fig. 6A). A second action potential could, however, be easily elicited during the negative afterpotential by means of a short hyperpolarization (fig. 6B). A hyperpolarizing pulse of constant amplitude and duration 


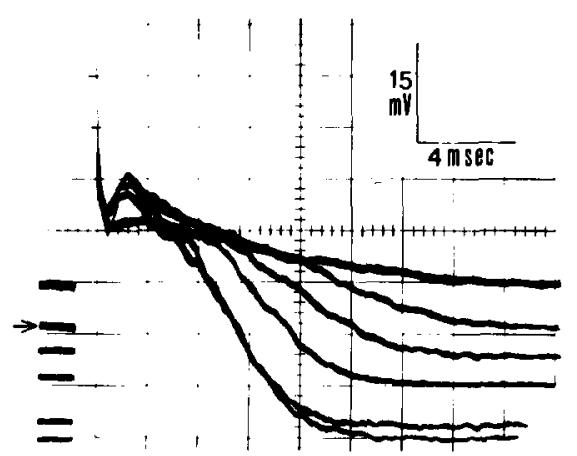

Fig. 5. Time course of decay of the negative afterpotential at different membrane potentials in a node treated with $5 \times$ $10^{-4} \mathrm{M}$ DDT for $55 \mathrm{~min}$. Arrow indicates normal resting potential. Sensitivity too high to record action potentials.

applied at various moments during the negative afterpotential induced at first and during the initial part of the afterpotential, a small graded response. The amplitude of this response increased as the delay between the first action potential and the hyperpolarizing pulse increased until finally a second action potential was induced. With further increase in delay the amplitude of the second action potential increased until it had the same amplitude as the first action potential. However, during the terminal phase of the negative afterpotential the hyperpolarizing pulse failed again to induce an action potential. Its only effect was a faster rate of decay of the remaining part of the afterpotential. Such anode break excitations could also be elicited during the initial part of the negative afterpotential with pulses of higher intensity.
They were also observed in the nodes in which DDT caused a prolongation of the whole falling phase of the action potential (fig. 6C). Abolition of the action potential or of the negative afterpotential was possible, but only with hyperpolarizing pulses of either long duration or very high amplitude. Anode break excitations were not observed in DDT-treated nodes which were in the resting state.

Finally, several single nodes were exposed to dieldrin in concentrations of up to $10^{-3} \mathrm{M}$. The only effect that could be observed was a slight prolongation of the falling phase of the action potential, even after more than $2 \mathrm{hr}$ of treatment with dieldrin. In one node dieldrin caused a more substantial prolongation of the action potential, and the duration of the action potential was increased to about 1.5 times normal. This extra-prolongation, however, was easily reversed by washing with fresh Ringer solution. Dieldrin had no effect on the resting potential.

\section{DISCUSSION}

The data presented in the first part of the Results show that DDT induces repetitive aetivity in only sensory fibres. Afferent nerves innervating skin, muscle and the lateral-line organs are all affected by DDT. Motor nerves, i.c., the ventral spinal root, showed no sign of repetitive activity, even after prolonged exposure to high DDT concentrations. The motor branch to the head of the gastrocnemius muscle, however, showed a slight repetitive activity (fig. 1D). This is probably due to sensory fibres which are present in

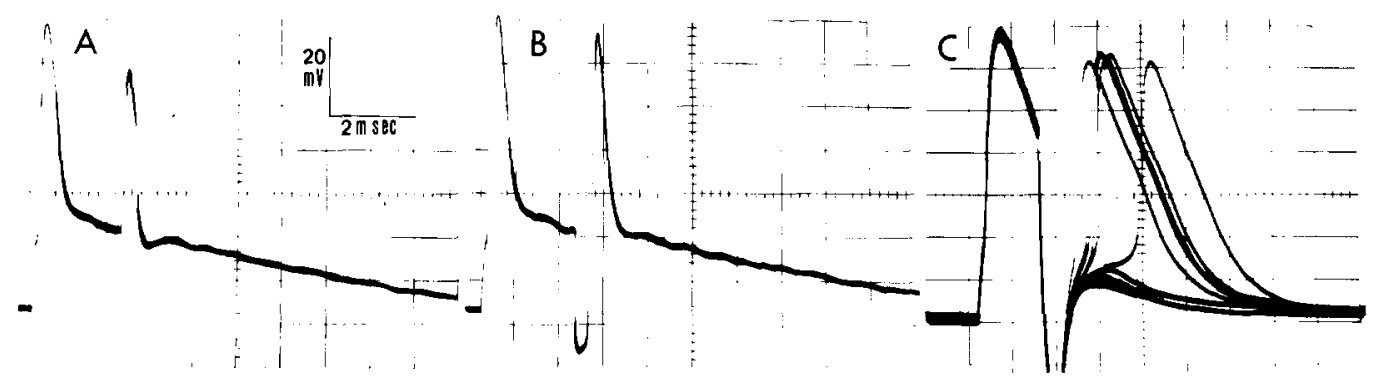

Fig. 6 . Effects of short current pulses applied during the negative afterpotential and the action potential in nodes treated with $5 \times$ $10^{-4} \mathrm{M}$ DDT. (A) A short depolarizing pulse applied during the first part of the negative afterpotential produces no active membrane response. (B) An anode break excitation elicited by a short hyperpolarizing pulse applied at the same instant. (C) Anode break excitations and abolition caused by a hyperpolarizing pulse of increasing amplitude applied during the prolonged falling phase of the action potential. 
this nerve branch, as can be easily demonstrated by centripetal recording. The peroneal and tibial branch are mixed nerves and therefore show an intermediate repetitive response after treatment with DDT (fig. 1B).

In DDT-poisoned rats too, repetitive activity only occurs in sensory fibres, as was revealed by experiments in which the sciatic nerve was excised with its spinal roots still attached ( $\mathrm{J}$. van den Bercken and $\mathrm{L}$. Sauren, unpublished observations).

The experiments with the single nerve fibres suggest that the negative afterpotential induced by DDT is probably directly responsible for the repetitive activity (fig. 2). Repetitive action potentials did not occur unless the negative afterpotential exceeded a critical level. Furthermore, the number of repetitive action potentials increased with increasing amplitude of the negative afterpotential, while the time interval between the action potentials decreased.

The repetitive activity in myelinated nerve fibres as described here differs from that in DDT-treated axons of the American cockroach. In these latter fibres, the number of repetitive action potentials did not increase gradually, but a train of spikes occurred abruptly when the negative afterpotential attained a certain level (Narahashi and Yamasaki, 1960). Since all axons showed a repetitive response after treatment with DDT, these authors suggested that DDT established a state in which every long-lasting depolarization caused repetitive activity. In myelinated nerve the situation is quite different, since a large negative afterpotential was frequently observed without any repetitive activity. There is probably a greater resemblance with DDT-poisoned lobster axons, in which the number of repetitive spikes and the amplitude of the negative afterpotential also increase progressively with time, and in which repetitive activity is sometimes absent (Narahashi and Haas, 1968).

The experiments with the single nodes of Ranvier show that DDT exclusively affects the falling phase of the action potential (fig. 4). Why DDT induced a distinct negative afterpotential in part of the fibres (fig. $4 \mathrm{C}, \mathrm{D}$ ), while in other fibres it caused a prolongation of the whole falling phase of the action potential (fig. 4E) is not known. Hille (1968) probably observed the same effects of DDT on the action potentials of frog nodes. Although he gave no figures of action potentials of DDT-treated nodes he stated that the prolongation of the action potential could sometimes be more appropriately described as a long, large negative afterpotential. From his voltage clamp data, Hille concluded that DDT specifically affected the sodium channels of the nerve membrane and caused a fraction of these channels which open during depolarization to close at a slower rate than is normal. DDT would not affect opening of the sodium channels, or sodium channels that were not open. The present results with DDT-poisoned nodes of Ranvier of Xenopus laevis are in substantial agreement with Hille's conclusions.

It is easy to account for the absence of any repetitive activity in DDT-treated nodes of fibres dissected from the sciatic nerve, both in Hille's study and in the present work, since only the large fibres were dissected all of which were probably motor fibres. However, the single nodes of the sensory fibres also failed to show repetitive activity, although they seemed more sensitive to DDT and subthreshold oscillations superimposed themselves on the negative afterpotential (fig. 4F). This illustrates once more that the negative afterpotential is not solely responsible for the repetitive activity, and that other important mechanisms are involved. The experiments with the hyperpolarizing pulses may throw some light on this question (fig. 6). In terms of the Hodgkin-Huxley equations, the main effect of such a short hyperpolarization is reversal of sodium activation and removal of sodium inactivation (Dodge, 1963). During the DDTinduced negative afterpotential there flows a persistent sodium current (Hille, 1968) which cannot be terminated by the hyperpolarizing pulse. This persistent sodium current exceeds threshold and an anode break excitation can occur because the hyperpolarizing pulse removes sufficient sodium inactivation. This implies that the voltage dependence of the sodium inactivation is not affected by DDT, as was actually shown by Hille (1968). Sodium inactivation is still high during the initial phase of the negative afterpotential and a much stronger hyperpolarization is needed to elicit a second action potential, while during the terminal phase of the afterpotential the persistent sodium current is too small to exceed threshold. The anode break excitations observed in the nodes in which DDT prolonge the whole falling phase of the action potential can be explained on the same basis. Abolition of the prolonged action potential is 
still possible, indicating that the persistent sodium current can be terminated by a strong hyperpolarization. This is also suggested by fig. 5 , which shows the dependence of the time course of decay of the negative afterpotential on the membrane potential. This figure further reveals that a decrease in steady-state inactivation of the sodium permeability does not produce a repetitive response; only a small depolarization appears as the membrane potential is made more negative.

These results suggest that only fibres which have a fast rate of recovery from inactivation may produce repetitive action potentials after treatment with DDT. That such a situation is more likely to occur in sensory fibres than in motor fibres is suggested by several experimental results. In contrast to motor fibres, sensory fibres often respond repetitively to a long-lasting depolarization and show a much slower rate of accomodation to linear rising currents (Schmidt and Stämpfli, 1964; Bergmann and Stämpfli, 1966). According to Vallbo (1964) and Frankenhauser and Vallbo (1965) this difference is mainly due to a faster rate of change of sodium inactivation in sensory fibres. On the other hand, the condition of the nerve fibres has a great influence on the rate of accomodation and hence, on the repetitive activity, as discussed by Honerjäger (1968). It is not surprising then that DDT should fail to induce repetitive activity in sensory fibres which are considered to have suffered damage during the dissection. The absence of repetitive activity which was sometimes observed in sciatic nerves excised from DDT-poisoned animals may also be due to the bad condition of the nerve as a result of the severe symptoms of poisoning.

The repetitive activity in afferent nerve fibres reported here will contribute to the changes in brain electrical activity in DDT-poisoned animals, as was tentatively concluded by Woolley and Barron (1968), and it may be at least partly responsible for the increased spinal reflex excitability (Dresden, 1949; Shankland, 1964; Isaacson, 1968). Moreover, a direct effect of DDT on sense organs can also result in repetitive afferent activity, as was recently shown for the lateral-line organ of Xenopus (van den Bercken and Akkermans, 1971). In addition, the marked prolongation of the action potential caused by DDT may result in an increase of the amount of transmitter released from the presynaptic nerve terminal upon excitation (Benoit and Mambrini, 1970). Thus there seems no doubt that with vertebrates as with insects, the peripheral nervous system is intimately involved in the DDT-poisoning syndrome.

Unlike DDT, dieldrin had almost no effect on myelinated nerve fibres of Xenopus laevis. The slight prolongation of the action potential mentioned here bears no relation to the severe symptoms of poisoning and repetitive activity or other effects were not observed in sciatic nerves excised from dieldrin-poisoned rats (van den Bercken, unpublished observations). Recently, it has been suggested that in the American cockroach, dieldrin is converted into aldrin-transdiol before it exerts its neurotoxic action (Wang et al., 1971). Whether the same is true for Xenopus and other vertebrates remains to be investigated.

\section{ACKNOWLEDGEMENTS}

I wish to thank Prof. H. van Genderen for his interest in this study and Dr. A.J. Kalmijn for his generous advice and invaluable help. This work was supported by grants from the Foundation of Fundamental Medical Research and from the Shell Corporation.

\section{REFERENCES}

Benoit, P.R. and J. Mambrini, 1970, Modification of transmitter release by ions which prolong the presynaptic action potential, J. Physiol. 210, 681.

Bercken, J. van den, 1968, The action of DDT and dieldrin on nerves and muscles of Xenopus laevis, Meded. Rijksfak. Landbouwwetensch. Gent 33, 1241.

Bercken, J. van den, 1969, The effect of DDT on single Ranvier nodes of Xenopus laevis, European J. Pharmacol. $9,146$.

Bercken, J. van den, and L.M.A. Akkermans, 1971, Negative temperature coefficient of the action of DDT in a sense organ, European J. Pharmacol. 16, 241.

Bergman, C. and R. Stämpfli, 1966, Difference de perméabilité de fibres nerveuses myélinisées sensorielles et motrices a l'ion potassium, Helv. Physiol. Acta 24, 247.

Dodge, F.A., 1963, A study of ionic permeability changes underlying excitation in myelinated nerve fibres of the frog. Thesis, The Rockefeller Institute, New York (University Microfilms, Inc., Ann Arbor, Michigan, No. 64-7333).

Dresden, D., 1949, Physiological investigations into the action of DDT. Thesis, University of Utrecht, The Netherlands. 
Eyzaguirre, C. and J.L. Lilienthal Jr., 1949, Veratrinic effects of Pentamethylenetetrazol (Metrazol) and 2,2-bis(pchlorophenyl) 1,1,1-trichloroethane (DDT) on mammalian neuromuscular function, Proc. Soc. Exptl. Biol. 70, 272.

Frankenhauser, B. and A.B. Vallbo, 1965, Accomodation in myelinated nerve fibres of Xenopus laevis as computed on the basis of voltage clamp data, Acta Physiol. Scand. 63. 1 .

Giannotti, O., R.L. Metcalf and R.B. March, 1956, The mode of action of Aldrin and Dieldrin in Periplaneta americana (L.), Ann. Entomol. Soc. Amer. 49, 588.

Hayes, W.J. Jr., 1959, Pharmacology and toxicology of DDT, in: DDT, the Insecticide Dichlorodiphenyltrichloroethane and its Significance, Vol. II, ed. P. Müller (Birkhäuser, Basel) p. 11.

Hille, B., 1968, Pharmacological modifications of the sodium channels of frog nerve, J. Gen. Physiol. 51, 199.

Honerjäger, P., 1968, Die repetitive Aktivität motorischer und sensibler markhaltiger Nervenfasern des Frosches, Pflügers Arch. 303, 55.

Isaacson, P.A., 1968, Action of DDT on the peripheral nervous system of the grass frog Rana pipiens, Curr. Sci. 18, 530.

Narahashi, T., 1971, Effects of insecticides on excitable tissues, in: Advances in Insect Physiology, Vol. 8, eds. J.W.L. Beament, J.E. Treherne and V.B. Wigglesworth (Acad, Press, London and New York) p. 1.

Narahashi, T. and H.G. Haas, 1968, Interaction of DDT with the components of lobster nerve membrane conductance, J. Gen. Physiol. 51, 177.

Narahashi, T. and T. Yamasaki, 1960, Mechanism of increase in negative afterpotential by dicophanum (DDT) in the giant axons of the cockroach, J. Physiol. 152, 122.

Nelson, P.G. and K. Frank, 1964, Extracellular potential fields of single spinal motoneurons, J. Neurophysiol. 27, 913.

Pichon, Y., 1969, Effets du DDT sur la fibre nerveuse isolée d'insecte. Etude en courant et en voltage imposés, J. Physiol. (Paris) 61, Suppl. 1, 162.

Roeder, K.D. and E.A. Weiant, 1946, The site of action of DDT in the cockroach, Science 103, 304.

Roeder, K.D. and E.A. Weiant, 1948, The effect of DDT on sensory and motor structures in the cockroach leg, J. Cell. Comp. Physiol. 32, 175.
Ryan, W.H. and D.L. Shankland, 1971, Synergistic action of cyclodiene insecticides with DDT on the membrane of giant axons of the American cockroach Periplaneta americana (L.), Life Sci. 10, 193.

Schmidt, H. and R. Stämpfli, 1964, Nachweis unterschiedlicher elektrophysiologischer Eigenschaften motorischer und sensibler Nervenfasern des Frosches, Helv. Physiol. Acta 22, C 143.

Shanes, A.M., 1951, Electrical phenomena in nerve. III. Frog sciatic nerve, J. Cell. Comp. Physiol. 38, 17.

Shankland, D.L., 1964, Involvement of spinal cord and peripheral nerves in DDT-poisoning syndrome in albino rats, Toxicol. Appl. Pharmacol. 6, 197.

Vallbo, Å.B., 1964, Accomodation related to inactivation of the sodium permeability in single myelinated nerve fibres from Xenopus laevis, Acta Physiol. Scand. 61, 429.

Wang, C.M., T. Narahashi and M. Yamada, 1971, The neurotoxic action of Dieldrin and its derivatives in the cockroach, Pesticide Biochem. Physiol. 1, 84.

Welsh, J.H. and H.T. Gordon, 1947, The mode of action of certain insecticides on the arthropod nerve axon, J. Cell. Comp. Physiol. 30, 147.

Woolley, D.E., 1968, Toxicological and pharmacological studies of visual and auditory potentials evoked in the cerebellum of the rat, Proc. West. Pharmacol. Soc. 11, 69 .

Woolley, D.E., 1970, Effects of DDT on the nervous system of the rat, in: The Biological Impact of Pesticides in the Environment, ed. J.W. Gillet, Environmental Science Series No. 1 (Oregon State University Press, Corvallis) p. 114

Woolley, D.E. and B.A. Barron, 1968, Effects of DDT on brain electrical activity in awake, unrestrained rats, Toxicol. Appl. Pharmacol. 12, 440.

Yamasaki, T. and T. Ishii, 1954, Studies on the mechanism of action of insecticides. VII. Activity of neuron soma as a factor of development of DDT symptoms in the cockroach, Botyu-Kagaku 19, 1. (English translation in: Japanese Contributions to the study of the insecticide-resistance problem (publ. by Kyoto University for W.H.O., 1957) p. 140).

Yamasaki, T. and T. Narahashi, 1958, Nervous activity as a factor of development of Dieldrin symptoms in the cockroach, Botyu-Kagaku 23, 47. 